\title{
Further Commentary on "Induced OBEs"
}

To the Editor:

I would like to make a small contribution to the discussion about out-of-body experiences (OBEs) allegedly induced by stimulating the angular gyrus. Some weeks ago, I read the case of a tinnitus patient who, his angular gyrus being stimulated during brain surgery, experienced himself as being " $50 \mathrm{~cm}$ behind his body and off to the 
left" (de Ridder, van Laere, Dupont, Menovsky, and de Heyning, 2007, p. 1830). He was unable voluntarily to control his disembodied location, and he did not see his physical body; in fact, he could see only from his physical body, not from the disembodied location (de Ridder, van Laere, Dupont, Menovsky, and de Heyning, 2007, p. 1830). The authors indicated clearly that the patient had his eyes open during the entire procedure.

Considering this "eyes open" condition, I recalled an earlier article about a Swiss patient who underwent similar brain stimulation during surgery to treat epilepsy (Blanke, Ortigue, Landis, and Seeck, 2002). Although the patient reported, "I see myself lying in bed, from above, but I only see my legs and lower trunk," because she was sitting at a 45 degree angle, if her eyes were open, the influence of physical vision might be an important factor to consider in understanding this perceptual phenomenon. As I was very curious, I emailed the senior author, Olaf Blanke, asking about the condition of the patient's eyes. Blanke kindly responded, "You ask an important question. The patient had her eyes open during the OBEs. But we tested this systematically, with closed eyes there was no OBE but the sensation of body movement" (O. Blanke, personal communication, November 20, 2007).

My understanding is that people have reported OBEs with both their eyes open and closed, that OBErs typically have visual perception from the disembodied location, that they usually see visual content that is not perceivable from the position of the physical body, and that unexpected perceptions have sometimes been investigated and shown to be accurate (Holden, Long, and MacLurg, 2006). By contrast, taking both brain stimulation cases together, at most, such stimulation has produced only the eyes-open sense of remote perception of erroneous visual content within physical visual range. Electrical brain stimulation has yet to produce (1) accurate vision of (2) material not visible to the physical eyes (3) from a remote location (4) with eyes closed - all typical features of spontaneous OBEs. I believe this information about the eyes-open condition of both patients adds one more point to Janice Holden, Jeffrey Long, and Jason MacLurg's (2006) critique of the report by Blanke and colleagues (2002).

\section{References}

Blanke O., Ortigue S., Landis T., and Seeck M. (2002). Stimulating illusory own-body perceptions: The part of the brain that can induce out-of-body experiences has been located. Nature, 419, 269-270. 
de Ridder D., van Laere K., Dupont P., Menovsky T., and de Heyning P. (2007). Visualizing out-of-body experience in the brain. New England Journal of Medicine, 357, 1829-1833.

Holden J., Long M. J., and MacLurg J. (2006). Out-of-body experiences: All in the brain? Journal of Near-Death Studies, 25, 99-107.

Iris Giesler-Petersen, Dipl. Jur. Hildesheimer-Straße 84 a 30169 Hannover, Germany e-mail: i.giesler@gmx.de 